Ann. Génét. Sél. anim., I970, 2 (2), III-II7.

\title{
INFLUENCE DE 'L'ALOPÉCIE SUR QUELQUES CARACTÉRISTIQUES BIOMÉTRIQUES DE LA SOURIS
}

\author{
N. KOBOZIEFF, F: REYNES et Genia GEMAHLING \\ Laboratoire de Génétique, Ecole nationale vétérinaire, 94-Alfort
}

\section{RÉSUMÉ}

L'alopécie chez la souris, symbole "alp ", est une anomalie récessive, non liée au sexe. Les sujets étudiés ont un aspect normal pendant les deux premières semaines après la naissance. Pendant toute la durée de la chute des poils, qui commence vers le 14 e jour, débute aux orbites et s'étend toujours d'avant en arrière, les sujets accusent un réel état de souffrance qui se caractérise par un arrêt de croissance et une perte de vitalité. Au 21 e jour, milieu de la dépilation, on observe une différence de poids significative entre les mâles alopéciques et les mâles normaux d'une même portée. Chez les femelles cette différence n'apparait aussí nettement que vers le $30^{\mathrm{e}}$ jour, toujours en faveur des sujets normaux. Au $60^{\mathrm{e}}$ jour après la naissance, l'ordre des moyennes est à nouveau celui rencontré au début de la dépilation, mais si les mâles alopéciques ont rattrapé le poids des mâles normaux, les femelles alopéciques elles, ne rattraperont le poids de leurs sœurs normales que vers l'âge de 3 mois. Il faut souligner que les mâles pèsent toujours plus lourd que les femelles, qu'il s'agisse de sujets alopéciques ou normaúx.

Enfin, notons que d'unè manière générale, les souris alopéciques sont plus fragiles que les sujets normaux et très sensibles au froid, surtout les femelles, qui, de plus, ne peuvent pas allaiter leurs pẹtits. Le taux de mortalité des sujets alopéciques est nettement plus important que celui des sujets normaux de la même lignée $(16,48 \%$ contre $5,8 \%$ ).

\section{INTRODUCTION}

L'alopécie chez la souris, symbole " alp", est une anomalie de la peau, récessive, non liée au sexe (КоBozIEFF et al., I958). Les sujets atteints ont un aspect normal pendant les deux premières semaines après la naissance. La chute des poils débute aux orbites et s'étend toujours d'avant en arrière, tout en respectant relativement longtemps les oreilles. La souris apparaît " alopécique "; c'est-à-dire complètement nue vers $1 \mathrm{a} 3-4^{\mathrm{e}}$ semaine. On observe alors d'autres phénomènes : épaississement et changement de couleur de la peau, formation de kystes et par- 
fois de verrues, allongement des griffes. Les plis transversaux apparaissent d'abord derrière la nuque, puis ils gagnent le front en mosaïque, enfin tout le corps se couvre de rides ondoyantes et donnent à l'animal un aspect très caractéristique (fig. I). La vie des sujets alopéciques est, en général, de courte durée : dans les conditions de notre laboratoire ils dépassent rarement 7 à 8 mois tandis qu'une souris normale vit en moyenne I6 mois. A l'autopsie on constate l'absence de dépôts de graisse et souvent une dégénérescence du foie.

Les anomalies de la peau, et plus particulièrement l'alopécie, ont fréquemment une influence néfaste sur le développement des sujets atteints. Ce fait a été signalé par différents auteurs ayant étudié diverses espèces. Nous n'en citerons que quelques-uns : ELDRIDGE et al. (I953), qui décrit une absence partielle, en bandes, de poils chez les bovins, Popova-Wassina (I93I), MoHR (r928), JohNson (r944), qui ont étudié diverses formes d'alopécie chez le mouton.

Ces différentes observations demandaient confirmation et le but de cette étude est de faire ressortir l'influence de l'alopécie sur quelques caractéristiques biométriques : mortalité et croissance pondérale ${ }^{(\mathbf{1})}$.

\section{MATÉRIEL ET MÉTHODE}

Les souris alopéciques étudiées ici, sont issues de croisements entre mâle alopécique homozygote et femelle normale hétérozygote. Pour les croisements réciproques nous avons rencontré de grandes difficultés : les glandes mammaires des femelles alopéciques sont peu développées et les petits, enlevés à leur mère dès la naissance, devaient être confiés à une nourrice de lignée normale. Aussi les effectifs dont nous disposions sont trop faibles pour être pris en considération, d'autant plus qu'ils risquaient de faire intervenir d'autres facteurs (lait maternel, etc...). lieu :

Les pesées ont été effectuées au dixième de gramme près, entre 11 heures et 12 heures et ont eu

- à la naissance,

- au début de la dépilation (14 et $15^{\mathrm{e}}$ jour),

- au milieu de la dépilation (21 e jour - période de sevrage),

- à la dépilation complète (30 jours - âge adulte),

- au $60^{\mathrm{e}}$ jour (les croisements sont effectués après 1 mois),

- au $90^{e}$ jour, en pleine maturité.

Au point de vue méthodologique, nous avons utilisé le test $\chi^{2}$, l'analyse de la variance à une voie et, quand le test $\mathrm{F}$ était significatif, nous avons comparé les poids moyens deux à deux par test de comparaison de moyennes ordonnées de Duncan (1955). Les effectifs étant différents d'un échantillon à l'autre, nous avons adopté la méthode de KRAmER (1956), simple extension du test précédent.

\section{RESSULTATS}

\section{Étude de la mortalité}

Dans le tableau I, où sont rassemblés les résultats concernant la mortalité, ne figurent pas $I 7$ sujets morts avant le début de la dépilation. Pour ces sujets, il était en effet impossible de connaître la proportion de ceux qui auraient été alopéciques : ils ont donc été écartés de l'étude.

I) Une note préliminaire a été présentée à la Journée de Génétique animale du 28 nov. I968 (ref. KOBOZIEFF, I969). 


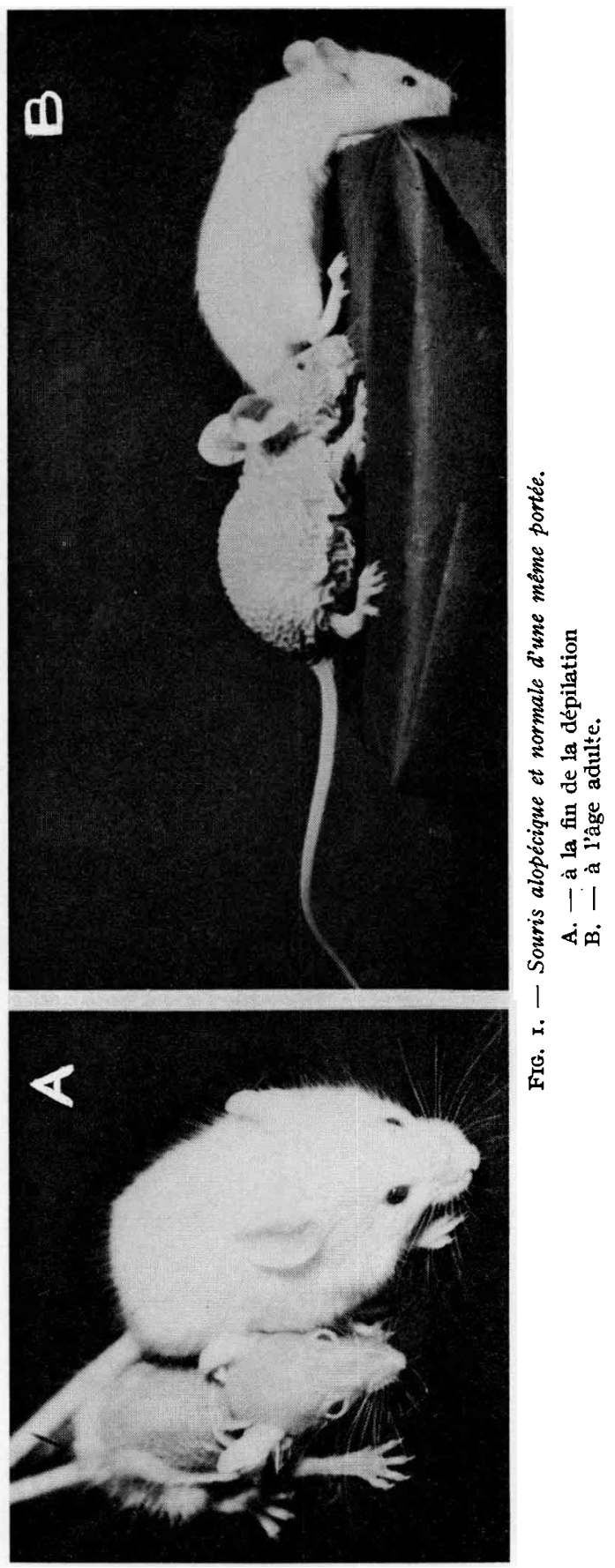


TABLEAU I

Mortalité suivant le sexe et la qualité des individus

\begin{tabular}{|c|c|c|c|c|c|}
\hline & \multicolumn{2}{|c|}{ Mâles } & \multicolumn{2}{|c|}{ Femelles } & \\
\hline & normaux & alopéciques & normales & apéciques & Total \\
\hline 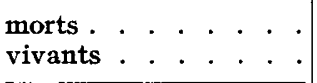 & $\begin{array}{r}3 \\
40\end{array}$ & $\begin{array}{r}7 \\
37\end{array}$ & $\begin{array}{r}1 \\
25\end{array}$ & $\begin{array}{r}8 \\
39\end{array}$ & $\begin{array}{r}19 \\
141\end{array}$ \\
\hline Total . . . . . . & 43 & 44 & 26 & 47 & 160 \\
\hline
\end{tabular}

Si l'on examine le taux de mortalité suivant le sexe et la qualité des individus, on observe qu'il n'y a pas de différence significative : $\chi_{3}^{2}=4,46$. Il en est de même si l'on tient compte seulement du sexe de 1'individu : II,49\% des mâles contre $12,33 \%$ des femelles $\left(\chi_{1}^{2}=0,07\right)$. Par contre, il y a statistiquement plus de sujets alopéciques qui meurent $(16,48 \%)$ que de sujets normaux $(5,8 \%)$ : $\chi_{1}^{2}=4,28$. On ne met pas de différence significative en évidence si l'on tient compte du sexe pour ces deux catégories d'individus. Notons cependant qu'ici les effectifs sont faibles et que, par conséquent, une différence même importante peut très bien n'être pas significative.

\section{Étude de la croissance pondérale}

Les I6o sujets ont été répartis en quatre catégories suivant deux critères : sexe et qualité de 1'individu. Ayant marqué, avant l'expérience, tous les sujets, nous appelons mâle " alp " (respectivement femelle "alp ") les mâles (respectivement les femelles) qui subiront la dépilation.

TABLEAU 2

Résultats de l'analyse de la variance des poids moyens pour les six stades de développement

\begin{tabular}{|c|c|c|c|c|c|c|}
\hline & naissance & $\begin{array}{l}\text { début de la } \\
\text { dépilation }\end{array}$ & $\begin{array}{l}\text { milieu } \\
\text { de la } \\
\text { dépila- } \\
\text { tion }\end{array}$ & $\begin{array}{c}\text { dépila- } \\
\text { tion } \\
\text { complète }\end{array}$ & $\begin{array}{c}60 \text { jours } \\
\text { après la } \\
\text { nais- } \\
\text { sance }\end{array}$ & $\begin{array}{l}90 \text { jours } \\
\text { après la } \\
\text { nais- } \\
\text { sance }\end{array}$ \\
\hline $\begin{array}{l}\text { Variance facto- } \\
\text { rielle. } \\
\text { Degrés de liberté }\end{array}$ & 0,203 & $\begin{array}{c}4,084 \\
3\end{array}$ & $\begin{array}{c}25,335 \\
3\end{array}$ & $\begin{array}{c}154,499 \\
3\end{array}$ & $\begin{array}{c}315,992 \\
3\end{array}$ & $\begin{array}{c}\text { I } 89,976 \\
3\end{array}$ \\
\hline $\begin{array}{l}\text { Variance rési- } \\
\text { duelle } \cdot \text {. } \\
\text { Degrés de liberté }\end{array}$ & $\begin{array}{l}0,070 \\
\text { I55 }\end{array}$ & $\begin{array}{l}0,963 \\
\text { I } 55\end{array}$ & $\begin{array}{c}2,972 \\
129\end{array}$ & $\begin{array}{c}5,610 \\
135\end{array}$ & $\begin{array}{c}6,723 \\
132\end{array}$ & $\begin{array}{c}7,277 \\
\mathbf{1} 29\end{array}$ \\
\hline $\begin{array}{l}\text { Valeur de } \mathbf{F} . \\
\text { Signification } \\
.\end{array}$ & $2,5 \% \stackrel{2,900}{<} \stackrel{\mathrm{P}<5 \%}{2}$ & I $\%<\stackrel{4,240}{\mathrm{P}<}<$ I $\%$ & $P \stackrel{8,524}{<}$ I $\%$ & $\begin{array}{c}27,533 \\
P \ll I\end{array}$ & $\begin{array}{c}47,001 \\
\mathrm{P} \ll \\
\ll\end{array}$ & $\begin{array}{l}26,106 \\
P \ll I \%\end{array}$ \\
\hline
\end{tabular}


Le tableau 2 donne les résultats de l'analyse de la variance des poids moyens pour les six stades du développement considérés.

Quelle que soit la période considérée, le $\mathrm{F}$ est significatif. Dans ces conditions 1a recherche de sous groupes homogènes de moyennes a été faite et les résultats sont inscrits dans le tableau 3. Les différences entre poids moyens peuvent venir, du sexe de l'individu d'une part et de la qualité de l'individu d'autre part. On sait qu'en général, les mâles pèsent plus que les femelles. C'est bien ce que l'on observe dans tous les cas, en ce qui concerne tout au moins les mâles normaux.

TABLEAU 3

Résultats du test de Duncan-Kramer pour les comparaisons multiples des poids moyens suivant la période d'observation

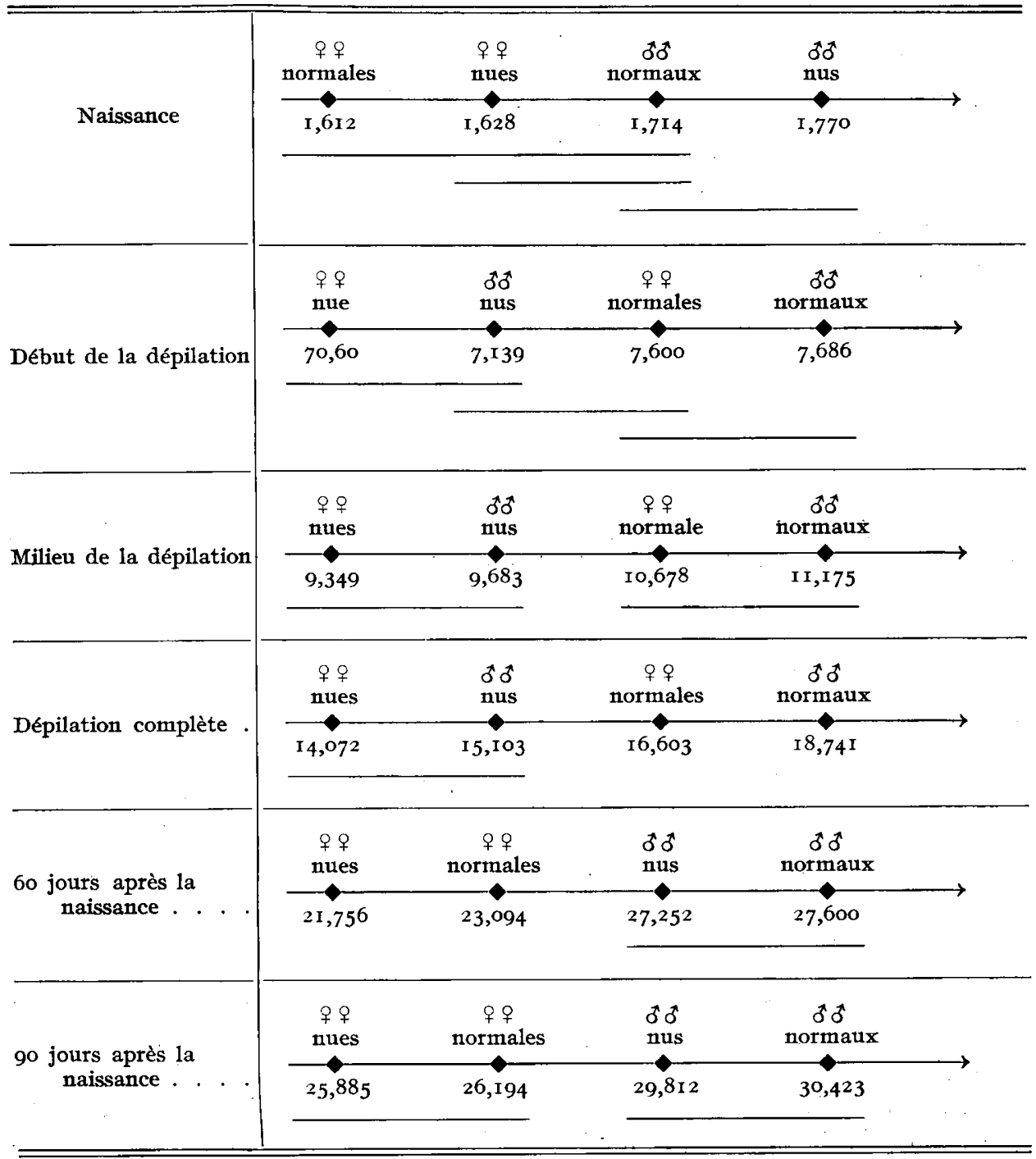


Par contre, en ce qui concerne les mâles alopéciques, il n'en va pas de même. Dès la naissance, on observe une différence significative entre les divers poids moyens. Cette différence s'accentue au début de la dépilation et devient très nette au milieu de ce processus. A cette époque, on peut distinguer deux sousgroupes homogènes, l'un constitué par les sujets alopéciques, l'autre par les sujets normaux. Cette situation se maintient à la dépilation complète, avec cependant une modification : le mâle normal a un poids moyen supérieur à celui de la femelle normale. Dans la période suivante, le mâle alopécique a déjà regagné le retard de poids pris pendant la période de souffrance; il n'en est pas de même pour la femelle alopécique dont le poids moyen est inférieur à celui de la femelle normale. Enfin, à go jours après la naissance, les effets de la dépilation se sont estompés aussi bien pour les mâles que pour les femelles et 1'on observe seulement une différence pondérale entre mâles et femelles.

La dépilation agit donc sur la croissance pondérale en provoquant un ralentissement, voire un arrêt de croissance. Ce dernier s'opère d'une façon différentielle suivant le sexe de l'individu. Les femelles mettent plus de temps que les mâles à retrouver le poids de leurs frères et sœurs. La mortalité différentielle observée suivant la qualité du sujet pourrait donc s'expliquer, tout au moins en partie, par cet arrêt de croissance : les souris qui perdent trop de poids meurent.

\section{DISCUSSION}

L'alopécie partielle ou totale est relativement fréquente chez la souris. Nous avons passé en revue, dans nos précédents articles, les principales études qui en ont été faites, KовоzIEFF et al. (1958, r964). Cependant, peu d'auteurs donnent des indications précises sur le développement des sujets atteints. EBbENHorst Tengbergen (I939), signale que les souris $N / N$ (naked) ont, à la naissance, le même aspect et poids que leurs frères et sœurs normaux; ils perdent rapidement du poids à partir de l'âge de 5-Io jours, bien avant le début de la dépilation, ceux qui survivent atteignent rarement plus des $3 / 4$ de la taille normale. De même, GARBER (I952), mentionne que les souris $h r^{b a}$ (bald), dès le I $4^{\mathrm{e}}$ jour pèsent moins lourds que les sujets normaux de la même portée. Ces deux anomalies sont phénotypiquement assez semblables à celle que nous avons étudiée, mais à notre connaissance, aucun auteur n'a entrepris une étude systématique de l'influence de l'anomalie sur le développement pondéral des sujets atteints.

Nous-mêmes, au cours de cette étude, avons rencontré de grandes difficultés dues au fait que la vitalité des souris alopéciques est, en général, compromise : la mortalité des souris atteintes d'alopécie est statistiquement plus élevée que celle des sujets normaux de la même portée et leur croissance pondérale est perturbée. Les souris atteintes d'alopécie qui perdent trop de poids meurent, tandis que celles qui luttent et augmentent de poids à la fin de la chute des poils, repren- 
nent le poids de leurs frères et sœurs normaux. Cette reprise de poids s'opère de façon différente suivant le sexe de l'individu : ce sont d'abord les mâles alopéciques qui rattrapent le poids de leurs frères normaux. Cependant, leur vie elle-même est, dans la majorité des cas de courte durée : 6 à 8 mois. La durée de puissance sexuelle des mâles est souvent réduite.

Reçu pour publication en février 1970

\section{SUMMARY}

\section{EFFECT OF ALOPECIA ON SOME BIOMETRIC CHARACTERISTICS IN THE MOUSE}

Alopecia in the mouse ( $A$ Alp $*$ symbol) is a reccessive abnormality which is not sex-linked. The subjects studied have a normal aspect during the first 2 weeks after birth. They begin to lose their coat around the 14th day, beginning at the eye sockets and extending towards the back. During this whole period, the subjects suffer acutely. Growth stops and vitality is diminished. On the 21 st day in the middle of this period, a signifiant weight difference is observed between alopecic males and normal males of the same litter. In the female, this difference (always in favor of normal subjects) does not become apparent until about the 30th day. At 60 days after birth, the average is again the same as that at the beginning of depilation, but while alopecic males recover their normal weight, alopecic females only recover this weight at around $\mathbf{3}$ months. It should be noted that alopecic or normal males always weigh more than the females.

Finally, it should be remarked that alopecic mice are more frail than normal subjects, and more sensitive to the cold. This is especially true of females which cannot suckle their young. The mortality rate of alopecic subjects is clearly higher than that of normal subjects of the same strain (16.48 \% against $5.8 \%$ ).

\section{RÉFÉRENCES BIBLIOGRAPHIQUES}

Ebrenhorst Tengbergen W.J.P.R. van, 1939. Lethality and abnormal condition of the pelago of the Latvian mouse (dominant Naked). Genetica, 21, 369-385.

EIDRIDGE F.E. et ATKEson F.W., 1953. Streaked hairlessness in Holstein-Friesian cattle. A sex linked, lethal character. J. Hered., 44, 6, 265-271.

GARBER E.D., 1952. "Bald" a second allele of hairless in the house mouse. J. Hered., 18, I, 45-46. Jomsson D.H., r944. Quintuple lambs of woolless domestic Sheep. J. Hered., 35, $25 \mathrm{r}$.

Kobozieff N., Pomriaskinsky-Kobozieff N., Drieux H., r958. Alopécie héréditaire associée à d'autres affections chez la souris. Note préliminaire. Rec. Méd. Vét., 134, 375-384.

Koboziefr N., Pomriaskinsky-Koboziefr N., Gemahring E., I964. Etude génétique sur l'alopécie héréditaire chez la souris. Ass. Fr. Rech. Phys. et Path. Cut., 30-34.

Ковоzпғғ N., 1969. Etude pondérale du développement des souris alopéciques. Ann. Génét. Sél. Anim., 1, I80.

KRAMER, 1956. Extension of multiple range tests to group means with uniqual numbers of replications. Biometrics, 12, 307-3ro.

MOHR J., I928. Hairless, a new recessive lethal in cattle. J. Genetics, 19, 3I5-336.

Popova-Wassina E.T., 1931. A naked lamb. J. Hered., 22, 91. 